\section{ASBESTOSIS AND EXPOSURE LEVELS IN A CHINESE ASBESTOS WORKER COHORT}

Xiaorong Wang, Midori N Courtice, Sihao Lin, Hong Qiu, Ignatius TS Yu. The Chinese University of Hong Kong, Hong Kong, China

10.1136/oemed-2014-102362.178

Objectives To assess the relationship between quantitative exposure levels and the development of asbestosis in a Chinese asbestos worker cohort.

Method A cohort consisting of 577 male workers from an asbestos products factory in China was followed for 37-years. Personal information was collected, including date of hire, specific job types, duration of exposure, and smoking habits. There were 127 workers $(22 \%)$ diagnosed as asbestosis by a specialised panel using Chinese radiographic Diagnostic Criteria for Pneumoconioses (GB5908-86). Individual cumulative fibre exposures (f$\mathrm{yrs} / \mathrm{ml}$ ) were estimated based on periodic dust/fibre measurements from different workshops and years of working at specific workshops, and then categorised into four levels (quartile). The relationship between the exposure levels and cumulative incidence of asbestosis was assessed with Cox Proportional Hazard Model, adjusting for age and smoking.

Results Workers at the four exposure levels were comparable in age at entry, exposure duration (around $25 \mathrm{yrs}$ ), and smoking rate. However, the proportion of asbestosis cases was grater with exposure levels, accounting for $9 \%, 27 \%, 29 \%$ and $36 \%$ from $1^{\text {st }}$ quartile to $4^{\text {th }}$ quartile, respectively. Hazard ratios for cumulative incidence of asbestosis showed a clear trend with the exposure levels, with a nearly three-fold increase $(3.42 .95 \%$ CI $2.0,5.9)$ at the highest exposure level, compared to at the lowest level.

Conclusions The study using quantitative estimate of exposures, which was seldom available in China, provides additional evidence for the exposure-response relationship between chrysotile exposure and the development of asbestosis in asbestos workers.

\section{THE IMPORTANCE OF CONDUCTING REGULAR SAFETY INSPECTIONS IN SMALL AND MEDIUM SIZE ENTERPRISES}

'Behdin Nowrouzi, ${ }^{1,2}$ Basem Gohar, ${ }^{3}$ Behnam Nowrouzi, ${ }^{3}$ Martyna Garbaczewska, ${ }^{4}$ Olena Chapovalov, ${ }^{5}$ Lorraine Carter. ' Laurentian University, Sudbury, Canada; ${ }^{2}$ Center for Research in Occupational Safety and Health, Sudbury, Canada; ${ }^{3}$ University of Toronto, Toronto, Canada; ${ }^{4}$ Public Services Health \& Safety Association, Toronto, Canada; ${ }^{5}$ Northern Ontario School of Medicine, Sudbury, Canada; ${ }^{6}$ Nipissing University, North Bay, Canada

\subsection{6/oemed-2014-102362.179}

Objectives A considerable effort has been made to examine the health and safety of employees in large-sized enterprises. However, there has not been much attention given to the organisation of work, occupational health and safety, and work disability prevention in small and medium enterprises (SME). The purpose of our study is to examine facilitators and barriers to occupational health and safety among SME in Ontario.

Method A cross-sectional design was used to examine the occupational health and safety culture of small and medium sized enterprises from public and private sectors in Ontario. A convenience sample of employees from all position titles in Ontario organisations that ranged from 5 to 100 full-time equivalent employees were invited via email to participate in the survey.

Results A total of a 153 questionnaires were returned. Most of the respondents were female $(84.2 \%)$ with a mean age of 49.8 years $(S D=10.6)$. Multivariable logistic regression modelling revealed the odds of a safe work environment for SME who conducted regular safety inspections were estimated to be 2.88 (95\% CI, 1.57-5.27) greater than the odds of a safe work environment for SME who did not conduct regular safety inspections.

Conclusions This study profiled the work and safety among small and medium enterprises in Ontario. Moreover, better implementation and training strategies that focus on adapting occupational health and safety legislation to the nature and diversity of SMEs is warranted.

\section{WORK ABILITY AND WORK-RELATED STRESS: A CROSS- SECTIONAL STUDY OF OBSTETRICAL NURSES IN URBAN NORTHEASTERN ONTARIO}

${ }^{1}$ Behdin Nowrouzi, ${ }^{1}$ Nancy Lightfoot, 'Lorraine Carter, 'Michel Lariviere, 'Ellen Rukholm, ${ }^{1}$ Robert Schinke, ${ }^{3}$ Diane Belanger-Gardner. 'Laurentian University, Sudbury, Canada; ${ }^{2}$ Nipissing University, North Bay, Canada; ${ }^{3}$ Health Sciences North, Sudbury, Canada

\subsection{6/oemed-2014-102362.180}

Objectives The aim of this study was to determine: 1) if quality of work life (QWL), location of cross-training, stress variables, and various demographic factors in nurses are associated with work ability, and 2) nursing occupational stress, QWL, and various associated factors are related with nurses' work ability.

Method This cross sectional study was conducted in 2012 in four hospitals in northeastern Ontario, Canada. A stratified random sample of registered nurses $(\mathrm{n}=111)$ were selected.

Results The majority of participants were female (94.6\%) ranging in age from 24 to 64 years $(M=41.9$, s.d. $=10.2)$. For the stress and QWL model, one variable: QWL (home-work support) $(p=0.015)$, cross-trained nurses $(p=0.048)$, and having more than 4 patients per shift $(\mathrm{p}=0.024)$ significantly contributed to the variance in work ability scores. In the logistic regression model, the odds of a higher work ability for nurses who received home-work support were estimated to be 1.32 (95\% CI, 1.06 to 1.66 ) times the odds of a higher work ability for nurses who did not receive home-work support.

Conclusions Work ability in the work environment of obstetrical nursing is important. To be high functioning, workplaces should maximise the use of their employees' actual and potential skills.

\section{SALIVARY CORTISOL RESPONSE TO A HIGH-PROTEIN CHALLENGE AND METABOLIC SYNDROME IN POLICE OFFICERS}

${ }^{1}$ Penelope Baughman, ${ }^{1}$ Michael Andrew, ${ }^{1}$ Cecil Burchfiel, ${ }^{1}$ Desta Fekedulegn, 2John Violanti, 'Diane Miller. 'National Institute for Occupational Safety and Health, Morgantown, WV, USA; ${ }^{2}$ School of Public Health and Health Professions, University at Buffalo, Buffalo, NY, USA

\subsection{6/oemed-2014-102362.181}

Objectives Policing is considered a high-stress occupation and officers have elevated cardiovascular morbidity and mortality. We evaluated the association between salivary cortisol response to a standardised challenge and the metabolic syndrome (MetSyn), an indicator of increased cardiovascular risk.

Method Cross-sectional data from the Buffalo Cardio-Metabolic Occupational Police Stress Study (2004-2009) were analysed. MetSyn was defined as three or more of five components: abdominal obesity, hypertension, elevated triglycerides, reduced high-density lipoprotein cholesterol, and glucose intolerance. Officers provided five salivary cortisol samples, one before challenge (ingestion of a high-protein shake) and four at 15-minute 
intervals thereafter, where increase represents normal response. Age- and sex-adjusted regression models were used to examine trends in mean number of components across quartiles of area under the curve (AUC) salivary cortisol. Patterns of mean cortisol response were assessed by MetSyn status using repeatedmeasures analysis of covariance.

Results The study included 373 officers $(74.0 \%$ men) with a mean age of 41.0 years. Prevalence of MetSyn was $25.7 \%$. The mean count of MetSyn components decreased (1.89, 1.75, 1.55, $1.37 ; P<0.01)$ across increasing quartiles of AUC salivary cortisol. The pattern of mean salivary cortisol decreased from baseline $(5.55,4.58,4.47,4.79,4.75 \mathrm{nmol} / \mathrm{L})$ in officers with MetSyn and increased $(5.08,5.82,5.92,5.82,5.60 \mathrm{nmol} / \mathrm{L})$ in their counterparts. The test for interaction between MetSyn status and sample timing was statistically significant $(P<0.001)$.

Conclusions A reduced cortisol response to a high-protein challenge may be associated with MetSyn. Future longitudinal studies could provide useful evidence for planning intervention studies on cardiovascular risk among police officers.

\section{AN EPIDEMIOLOGY OF TRAFFIC ACCIDENT IN NORTHERN THAILAND}

Tawatchai Apidechkul. Mae Fah Luang University, Chiang Rai, Thailand

\subsection{6/oemed-2014-102362.182}

Objectives A cross-section study design aimed to explain the characteristics of traffic accident cases in northern of Thailand.

Method A cross-section study design aimed to explain the characteristics of traffic accident cases in northern of Thailand. The subjects were recruited from 8 hospitals and 7 police stations in the highest case accident areas from hospitals and police stations during 2009- 2012. All medical records and police statements were used as the sources of data collection. All research procedures were approved by the committee for the protection of human subjects of Mae Fah Luang University.

Results Totally 35925 cases had been recruited into the study from 8 hospitals during 2009-2011. 66.58\% were female, $28.01 \%$ aged $16-25$ years old, and main vehicle was motorcycle (78.85\%). $29.31 \%$ of subjects had been admitted at the hospital, $77.06 \%$ had a length of admission $\leq 31$ days, $2.39 \%$ diability found, $84.81 \%$ had medical cost $\geq 5000$ baht. Univariate analysis found alcohol used, types of vehicle, medical cost, and medical surgery were statistical different between sex.

In 7 police stationts, 2339 cases had been recruited into the analysis. $71.43 \%$ were females, $27.61 \%$ were aged $16-25$ years old, $60.96 \%$ were married, $92.02 \%$ were Buddhism. The main type of vehicle was the cars $(56.60 \%), 67.29 \%$ had their accident on the highways, and $60.66 \%$ had accidents on the day time (06.00 am.-06.00pm.), and those $13.12 \%$ drank alcohol. Conclusions Thailand needs the specific study for improving driving safety behaviours and also an effective regulation to control for traffic accident.

\section{CONTRIBUTION TO THE PREVENTION OF OCCUPATIONAL STRESS}

Kandouci Chahrazed, Belhadj Zoubida, Baraka Fatiha, Kandouci Baderdine Abdelkrim. University of Sidi Bel-Abbes, Sidi Bel-Abbes, Algeria

10.1136/oemed-2014-102362.183
Objectives The objectives of this study were to assess the importance of the mental suffering of employees in the service sector of a city in western Algeria and to identify risk factors and moderators of this suffering in order to develop a effective prevention.

Method Standardised questionnaires were subjected to 753 employees in the tertiary sector. These questionnaires include three categories: A social and professional record (21 items), Maslach Burn Out Inventory: MBI (22 items), Job Personal Interaction Scale: JPIS 35 questions on the perception of the work environment by the individual divided into 6rubriques (workload and unpredictability, control, rewards, recognition and fairness at work, social support, conflicts and perceived value valuri work and training)

The data collected were anonymous and confidential. Data analysis was performed using SPSS (version 17.0). Univariate analysis was performed (chi-square Pearson correlations and a multivariate analysis (logistic regression).

Results The state of burn-out was $23.6 \%$ after logistic regression the main social determinants of psychological distress in the overall sample one taking medication to relieve pain $(\mathrm{p}=$ 0.0002) remained significant.

Regarding the determinants "professional" contact with the public and strong mode part of work were significant respectively ( $p=0.0017$ and 0.0042$)$.

Moderators of stress for the multivariate analysis recognised two subscales of bad training $(p<0.01)$ and conflicts of values and perceived value $(\mathrm{p}=0.02)$.

Conclusions Preventive actions to reduce or eliminate risk factors directly affect the environment and source of work: training more relevant, better organisation of work, especially the riding tasks and recognition of work done.

\section{EVALUATION OF THE PREVALENCE OF URINARY INCONTINENCE SYMPTOMS IN ADOLESCENT FEMALE SOCCER PLAYERS AND THEIR IMPACT ON QUALITY OF LIFE}

${ }^{1}$ Antonio Fernandes, ${ }^{1}$ Fatima Fitz, ${ }^{2}$ Alexandre Silva, ${ }^{3,1}$ Eduardo Filoni, ${ }^{3}$ José M Filho. ${ }^{1}$ University of Mogi Das Cruze, Mogi Das Cruzes, São Paulo, Brazil; ${ }^{2}$ Cruzeiro Do Sul University, São Paulo, São Paulo, Brazil; ${ }^{3}$ State University of Campinas, Campinas, São Paulo, Brazil

\subsection{6/oemed-2014-102362.184}

Objectives To evaluate the prevalence, reliability and severity of symptoms of urinary incontinence (UI) in adolescent female soccer players and the impact of those symptoms on quality of life. Method The present work is a cross-sectional study of 59 female adolescents divided into a group of athletes, which included 35 adolescent amateur soccer players aged between 12 and 19 years $(15.6 \pm 2.0)$, and a control group, which included 24 adolescents aged 11 to 19 years $(14.8 \pm 2.4)$ who did not practice any sports. The International Consultation on Incontinence Questionnaire - Short Form (ICIQ-SF), the pad test and King's Health Questionnaire (KHQ) were used for evaluation.

Results The athletes displayed $62.8 \%$ positivity the pad test and the ICIQ-SF, whereas the control group exhibited $25 \%$ positivity. A medium agreement of 0.45 was found between the applied evaluation scales, as assessed with Cohen's kappa coefficient. In terms of quality of life (as assessed with the KHQ), the athletes exhibited a score of 35.2 in the General Health domain, 37.3 in the Emotions domain and 26.5 in the Sleep/Energy domain. 